\title{
DILATIONS AND CONSTRAINED ALGEBRAS
}

\author{
Michael A. Dritschel, Michael T. Jury and Scott McCullough
}

Abstract. It is well known that contractive representations of the disk algebra are completely contractive. The Neil algebra $\mathscr{A}$ is the subalgebra of the disk algebra consisting of those functions $f$ for which $f^{\prime}(0)=0$. There is a complete isometry from the algebra $R(W)$ of rational functions with poles off of the distinguished variety $W=\left\{(z, w): z^{2}=w^{3},|z|<1\right\}$ to $\mathscr{A}$. We prove that there are contractive representations of $\mathscr{A}$ which are not completely contractive, and furthermore provide a Kaiser and Varopoulos inspired example of a representation $\pi$ of $R(W)$ whereby $\pi(z)$ and $\pi(w)$ are contractions, yet $\pi$ is not contractive. We also present a characterization of those contractive representations of $R(W)$ that are completely contractive. Finally, we show that by contrast, for the variety $\mathscr{V}=\left\{(z, w): z^{2}=w^{2},|z|<1\right\}$, all contractive representations of the algebra $R(\mathscr{V})$ of rational functions with poles off $\mathscr{V}$ are completely contractive, and we as well provide a simplified proof of Agler's analogous result over an annulus.

Mathematics subject classification (2010): Primary: 47A20; Secondary: 30C40, 30E05, 46E22, 46E25, 46E40, 46L07, 47A25, 47A48, 47L55.

Keywords and phrases: Dilations, inner functions, Herglotz representations, completely contractive representations, realizations, Nevanlinna-Pick interpolation.

\section{REFERENCES}

[1] Jim Agler, Rational dilation on an annulus, Annals of Math. (2) 121 (1985), no. 3, 537-563.

[2] JIM AGLER, private communication circa 1991.

[3] Jim Agler, John Harland and Benjamin J. Raphael, Classical function theory, operator dilation theory, and machine computation on multiply-connected domains, Mem. Amer. Math. Soc. 191 (2008), no. 892, viii+159 pp.

[4] Jim Agler, Greg Knese And John E. MCCARthy, Algebraic pairs of isometries, J. Operator Theory 67 (2012), no. 1, 215-236.

[5] Jim Agler and John E. McCarthy, Distinguished varieties, Acta Math. 194 (2005), no. 2, 133153.

[6] Jim Agler and John E. McCarthy, Parametrizing distinguished varieties, Recent advances in operator-related function theory, 29-34, Contemp. Math., 393, Amer. Math. Soc., Providence, RI, 2006.

[7] Jim Agler And John E. MCCARThy, Hyperbolic algebraic and analytic curves, Indiana Univ. Math. J., 56 (2007), no. 6, 2899-2933.

[8] William Arveson, Subalgebras of $C^{*}$-algebras, II, Acta Math. 128 (1972), no. 3-4, 271-308.

[9] Joseph A. Ball, Vladimir Bolotnikov, Sanne ter Horst, A constrained Nevanlinna-Pick interpolation problem for matrix-valued functions, Indiana Univ. Math. J. 59 (2010), no. 1, 15-51.

[10] Joseph A. Ball, Moisés Guerra Huamán, Test functions, Schur-Agler classes and transferfunction realizations: the matrix-valued setting, Complex Anal. Oper. Theory 7 (2013), no. 3, 529575.

[11] Joseph A. Ball, Moisés Guerra Huamán, Convexity analysis and matrix-valued Schur class over finitely connected planar domains, J. Operator Theory 70 (2013), no. 2, 531-571.

[12] STEVEN BELL, Finitely generated function fields and complexity in potential theory in the plane, Duke Math. J. 98 (1999) 187-207.

[13] SteVen Bell, A Riemann surface attached to domains in the plane and complexity in potential theory, Houston J. Math. 26 (2000) 277-297. 
[14] Adam Broschins Ki, Eigenvalues of Toeplitz Operators on the Annulus and Neil Algebra, Complex Anal. Oper. Theory 8 (2014), no. 5, 1037-1059.

[15] Kenneth R. Davidson, Vern I. Paulsen, Mrinal Raghupathi, Dinesh Singh, a constrained Nevanlinna-Pick interpolation problem, Indiana Univ. Math. J. 58 (2009), no. 2, 709-732.

[16] Michael A. Dritschel, James Pickering, Test functions in constrained interpolation, Transactions American Math. Society 364 (2012), no. 11, 5589-5604.

[17] Michael A. Dritschel, Scott McCullough, Test functions, kernels, realizations and interpolation, Operator theory, structured matrices, and dilations, 153-179, Theta Ser. Adv. Math., 7, Theta, Bucharest, 2007.

[18] Michael A. Dritschel and Scott McCullough, The failure of rational dilation on a triply connected domain, J. Amer. Math. Soc. 18 (2005), no. 4, 873-918.

[19] Maurice Heins, Extreme normalized analytic functions with positive real part, Ann. Acad. Sci. Fenn. Ser. A I Math. 10 (1985), 239-245.

[20] Michael T. Jury, Greg Knese, Scott McCullough, Nevanlinna-Pick interpolation on distinguished varieties in the bidisk, J. Funct. Anal. 262 (2012), no. 9, 3812-3838.

[21] Greg Knese, Polynomials defining distinguished varieties, Trans. Amer. Math. Soc. 362 (2010), no. $11,5635-5655$.

[22] Scott McCullough, Matrix functions of positive real part on an annulus, Houston J. Math. 21 (1995), no. 3, 489-506.

[23] Stephen Parrott, Unitary dilations for commuting contractions, Pacific J. Math. 34 (1970) $481-$ 490.

[24] Vern Paulsen, Completely bounded maps and operator algebras, Cambridge Studies in Advanced Mathematics, 78 Cambridge University Press, Cambridge, 2002. xii +300 pp.

[25] JAmes Pickering, Counterexamples to rational dilation on symmetric multiply connected domains, Complex Anal. Oper. Theory 4 (2010), no. 1, 55-95.

[26] Mrinal Raghupathi, Nevanlinna-Pick interpolation for $\mathbb{C}+B H^{\infty}$, Integral Equations Operator Theory 63 (2009), no. 1, 103-125.

[27] W. Rudin, Pairs of inner functions on finite Riemann surfaces, Trans. Amer. Math. Soc., 140 (1969), 423-434.

[28] N. Th. VAropoulos, On an inequality of von Neumann and an application of the metric theory of tensor products to operators theory, J. Functional Analysis, 16 (1974), 83-100.

[29] Prasada Vegulla, Geometry of distinguished varieties, Thesis (Ph. D.) - Washington University in St. Louis. 2007, 45 pp. 